\title{
The Inhibitory Effect of Rivastigmine and Galantamine on Choline Transport in Brain Capillary Endothelial Cells
}

\author{
Na-Young LeE, and Young-Sook KANG* \\ College of Pharmacy and Research Institute of Pharmaceutical Sciences, Sookmyung Women's University, Seoul 140-742, Republic of Korea
}

(Received January 11, 2010; Revised January 19, 2010; Accepted January 20, 2010)

\begin{abstract}
The blood-brain barrier (BBB) transport of acetylcholinesterase (AChE) inhibitors, donepezil and tacrine suggested to be mediated by choline transport system in our previous study. Therefore, in the present study, we investigated the interaction of other AChE inhibitors, rivastigmine and galantamine with choline transporter at the BBB. The effects of rivastigmine and galantamine on the transport of choline by conditionally immortalized rat brain capillary endothelial cell lines (TR-BBB cells) were characterized by cellular uptake study using radiolabeled choline. The uptake of $\left[{ }^{3} \mathrm{H}\right]$ choline was inhibited by rivastigmine and galantamine, with $\mathrm{IC}_{50}$ values (i.e. concentration necessary for $50 \%$ inhibition) for 1.13 and $1.15 \mathrm{mM}$, respectively. Rivastigmine inhibited the uptake of $\left.{ }^{3} \mathrm{H}\right]$ choline competitively with $K_{\mathrm{i}}$ of $1.01 \mathrm{mM}$, but galantamine inhibited noncompetitively. In addition, the efflux of $\left[{ }^{3} \mathrm{H}\right]$ choline was significantly inhibited by rivastigmine and galantamine. Our results indicated that the BBB choline transporter may be involved in a part of the influx and efflux transport of rivastigmine across the BBB. These findings should be therapeutically relevant to the treatment of Alzheimer's disease (AD) with AChE inhibitors, and, more generally, to the BBB transport of CNS-acting cationic drugs via choline transporter.
\end{abstract}

Keywords: Rivastigmine, Galantamine, Choline transport, Blood-brain barrier, TR-BBB cells

\section{INTRODUCTION}

Acetylcholinesterase (AChE) inhibitors, such as donepezil, tacrine, rivastigmine and galantamine, are clinically useful in the treatment of Alzheimer's disease (AD). The efficacy of $A D$ treatment drugs depends upon the ability of drugs to cross the blood-brain barrier (BBB) and reach therapeutic concentrations in brain following systemic administration. The BBB is formed by tight junctions of the brain capillary endothelial cells, which are highly restrictive for the entry of both large and small hydrophilic drugs used in the treatment of CNS disorder from the blood to the brain parenchyma cells (Pardridge, 1988). Actually, the clinical failures of most of the potentially effective therapeutics to treat the central nervous system disorders are often due to their poor permeability across the BBB (Ohtsuki and Terasaki, 2007). Therefore, the understanding of transport mechanism of these AChE inhibitors across the BBB would be important for therapeutic optimi-

${ }^{*}$ Corresponding author

Tel: +82-2-710-9562 Fax: +82-2-2077-7975

E-mail: yskang@sookmyung.ac.kr zation.

It was reported that the brain distribution of these AChE inhibitors such as donepezil and tacrine is relatively high (McNally et al., 1996; Matsui et al., 1999). The concentration of donepezil and tacrine in the brain was several times higher than those in the plasma. These findings suggested that carrier-mediated transport system(s) may be involved in the penetration of these drugs into the brain across the BBB. In our previous study, donepezil and tacrine competitively inhibited choline uptake via organic cation transporter 2 (OCT2) in conditionally immortalized rat brain capillary endothelial cell line (TR-BBB cells) (Kang et al., 2005). In addition, donepezil and tacrine inhibited choline efflux transport at the BBB (Lee and Kang, 2008). These findings indicated that this transporter may be also involved in the transport of basic drugs such as donepezil and tacrine across the BBB. Other reports also suggested that donepezil and tacrine were transported to the brain across the BBB by organic cation transporter (OCT) such as choline transport systems, CHT1 or OCT2 (Sung et al., 2005; Kim et al., 2009).

Rivastigmine and galantamine are also clinically avail- 
able drugs used for the treatment of $A D$ by the inhibition of AChE or butyrylcholinesterase (BChE) like donepezil and tacrine. These drugs are known to cross the blood-brain barrier, but the transport mechanism(s) remains unclear (Jann et al., 2002). Rivastigmine is a cabamate derivative, which has similar structure to acetylcholine and a molecular weight of 400.43. Galantamine is an alkaloid from the bulb and flowers of snowdrop, which is similar to codein. Since these AChE inhibitors are bulky organic cationic drugs in spite of widely differing molecular structures, organic cation transporter (OCT) including the choline transporter may be also involved in the transport of these drugs across the BBB like donepezil and tacrine. The BBB choline transporter has been proposed as a drug delivery system for various amine drugs such as eperisone, scopolamine and thiamine (Kang et al., 1990). Therefore, in the present study, we investigated the BBB transport mechanism of rivastigmine and galantamine by evaluation of the relationship between the choline transporter among the OCTs and these drugs using TR-BBB cells as an in vitro model of BBB.

\section{MATERIALS AND METHODS}

\section{Materials}

[Methlyl- $\left.{ }^{3} \mathrm{H}\right]$ Choline $\left(\left[{ }^{3} \mathrm{H}\right]\right.$ choline, $\left.82.0 \mathrm{Ci} / \mathrm{mmol}\right)$ was purchased from Amersham (Buckinghamshire, UK). Donepezil hydrochloride was kindly provided by Daewoong Co. (Seoul, Korea). Rivastigmine was provided from Novartis International Pharmaceutical Ltd. (Basel, Switzerland). Galantamine was purchased from Aldrich Chemical (St. Louis, MO, USA). All other chemicals and reagents were commercial products of reagent grade and were purchased from Sigma Chemical (St. Louis, MO, USA).

\section{Cell culture}

The TR-BBB cells were cultured with Dulbecco's modified Eagle's medium (Invitrogen, San Diego, CA, USA), supplemented with $10 \%$ fetal bovine serum, $100 \mathrm{U} / \mathrm{ml}$ penicillin, $100 \mu \mathrm{g} / \mathrm{ml}$ streptomycin (Invitrogen, San Diego, CA, USA) and $15 \mu \mathrm{g} / \mathrm{L}$ endothelial cell growth factor (Roche, Mannheim, Germany) at $33^{\circ} \mathrm{C}$ in a humidified atmosphere of $5 \% \mathrm{CO}_{2} /$ air. On rat tail collagen type I-coated 24 well culture plates (IWAKI, Tokyo, Japan) initial seeding was done at $1 \times 10^{5}$ cells/well for the uptake study. After incubation for 3 days at $33^{\circ} \mathrm{C}$, the cultures became confluent and then used in transport study.

\section{Transport studies in TR-BBB cells}

The $\left[{ }^{3} \mathrm{H}\right]$ choline uptake was performed according to the previous report (Kang et al., 2005). TR-BBB cells were washed three times with $1 \mathrm{ml}$ extracellular fluid (ECF) buffer consisting of $122 \mathrm{mM} \mathrm{NaCl}, 25 \mathrm{mM} \mathrm{NaHCO} 3,3 \mathrm{mM}$ $\mathrm{KCl}, 1.4 \mathrm{mM} \mathrm{CaCl}_{2}, 1.2 \mathrm{mM} \mathrm{MgSO}_{4}, 0.4 \mathrm{mM} \mathrm{K}_{2} \mathrm{HPO}_{4}, 10$ $\mathrm{mM}$ D-glucose and $10 \mathrm{mM}$ Hepes $(\mathrm{pH} 7.4)$ at $37^{\circ} \mathrm{C}$. Uptake was initiated by applying $200 \mu$ l containing $\left[{ }^{3} \mathrm{H}\right]$ choline $(28$ $\mathrm{nM}$ ) at $37^{\circ} \mathrm{C}$ in the presence or absence of $\mathrm{AChE}$ inhibitors. The cells were then solubilized by incubation overnight in $750 \mu \mathrm{l}$ of $1 \mathrm{~N} \mathrm{NaOH}$ at RT. An aliquot (50 $\mu$ l) was taken for quantification of cellular protein using a DC protein assay kit (Bio-Rad, Hercules, CA, USA) with bovine serum albumin as a standard. The remaining solution $(500 \mu \mathrm{l})$ was mixed with $5 \mathrm{ml}$ of scintillation cocktail (Hionic-fluor; Packard, Meriden, CT, USA) for measurement of radioactivity in a liquid scintillation counter (LS6500; Beckman, Fullerton, CA, USA).

The efflux of $\left[{ }^{3} \mathrm{H}\right]$ choline by TR-BBB cells was determined by incubating the cells for $60 \mathrm{~min}$ at $37^{\circ} \mathrm{C}$ with $\left[{ }^{3} \mathrm{H}\right]$ choline dissolved in ECF buffer. The medium was then removed, and the cells were washed with ice-cold ECF buffer. The incubation medium alone or containing inhibitors was added for desired times. After appropriate time periods, the incubation medium was removed and the amount of $\left[^{3} \mathrm{H}\right]$ choline remained in the cells was measured.

\section{Data analysis}

To analyze the competitive nature of rivastigmine and galantamine for $\left[{ }^{3} \mathrm{H}\right]$ choline, Lineweaver-Burk plots were generated. The inhibitory constant $\left(K_{i}\right)$ was calculated from equation (1):

$$
V=V_{\max } \cdot C /\left[K_{m} \cdot\left(1+\mathrm{l} / K_{\mathrm{i}}\right)+\mathrm{C}\right]
$$

where I corresponds to the concentration of rivastigmine and galantamine, respectively. $V, C, K_{m}$ and $V_{\max }$ are velocity of saturable uptake, the substrate concentration in the uptake medium, the Michaelis-Menten constant and the maximum velocity of uptake, respectively.

Statistical analyses were carried out by one-way ANOVA with Dunnett's post-hoc test.

\section{RESULTS}

First, the effects of rivastigmine and galantamine on choline influx transport in TR-BBB cells were studied by assessing the ability of these compounds to inhibit $\left[{ }^{3} \mathrm{H}\right]$ choline uptake. $\left[{ }^{3} \mathrm{H}\right]$ Choline uptake in the presence of $2.0 \mathrm{mM}$ rivastigmine and galantamine was decreased to about $30 \%$ of the control level (Table I). The inhibition of $\left[{ }^{3} \mathrm{H}\right]$ choline uptake caused by these drugs occurred in a 
concentration dependent manner.

The dose-response relationship for the inhibition of $\left[{ }^{3} \mathrm{H}\right]$ choline uptake by donepezil, rivastigmine and galantamine in TR-BBB cells is given in Fig. 1. In our previous report, it was already found that AChE inhibitors such as donepezil and tacrine inhibited choline uptake competitively (Kang et al., 2005). However, the $\mathrm{IC}_{50}$ value (i.e. concentration necessary for $50 \%$ inhibition) for donepezil was not calculated, so we also examined the inhibition of $\left[{ }^{3} \mathrm{H}\right] \mathrm{chol}-$ ine uptake by donepezil. $\left[{ }^{3} \mathrm{H}\right]$ Choline uptake was decreased by various $\mathrm{AChE}$ inhibitors in a dose-dependent manner. Donepezil inhibited $\left[{ }^{3} \mathrm{H}\right]$ choline uptake more strongly than rivastigmine and galantamine. It is indicated that donepezil is a more potent inhibitor of choline uptake than rivastigmine and galantamine. The $\mathrm{IC}_{50}$ values for donepezil, rivastigmine and galantamine were $0.068,1.13$ and $1.15 \mathrm{mM}$, respectively (Table II).

Furthermore, we investigated the kinetic nature of the inhibitory effect of rivastigmine and galantamine on choline uptake. Lineweaver-Burk plot of choline uptake in TR-BBB cells showed mutual inhibitory effect by rivastigmine and galantamine (Fig. 2). In the presence of $500 \mu \mathrm{M}$ riva-

Table I. Effect of rivastigmine and galantamine on the $\left[{ }^{3} \mathrm{H}\right]$ choline uptake in TR-BBB cells

\begin{tabular}{lcc}
\hline Substrate & $\begin{array}{c}\text { Concentration } \\
(\mathrm{mM})\end{array}$ & $\begin{array}{c}\text { Uptake of }\left[{ }^{3} \mathrm{H}\right] \text { choline } \\
(\% \text { of control) }\end{array}$ \\
\hline Control & & $100 \pm 3$ \\
Rivastigmine & 0.5 & $75.3 \pm 3.1^{\mathrm{a}}$ \\
Galantamine & 2.0 & $32.8 \pm 1.9^{\mathrm{b}}$ \\
& 0.5 & $78.2 \pm 1.9^{\mathrm{a}}$ \\
\hline
\end{tabular}

$\left[{ }^{3} \mathrm{H}\right]$ Choline uptake by TR-BBB cells was measured in the absence (control) or presence of rivastigmine and galantamine for $5 \mathrm{~min}$ at $37^{\circ} \mathrm{C}$. Each value represents the mean \pm S.E.M. $(n=3-4) .{ }^{a} p<0.05$, ${ }^{\mathrm{b}} p<0.001$; significantly different from control. stigmine the $K_{m}$ value for choline uptake by TR-BBB cells increased from 39.6 to $51.7 \mu \mathrm{M}$, whereas the $V_{\max }$ did not change significantly. These results indicated that rivastigmine competitively inhibited choline uptake with a $K_{\mathrm{i}}$ of

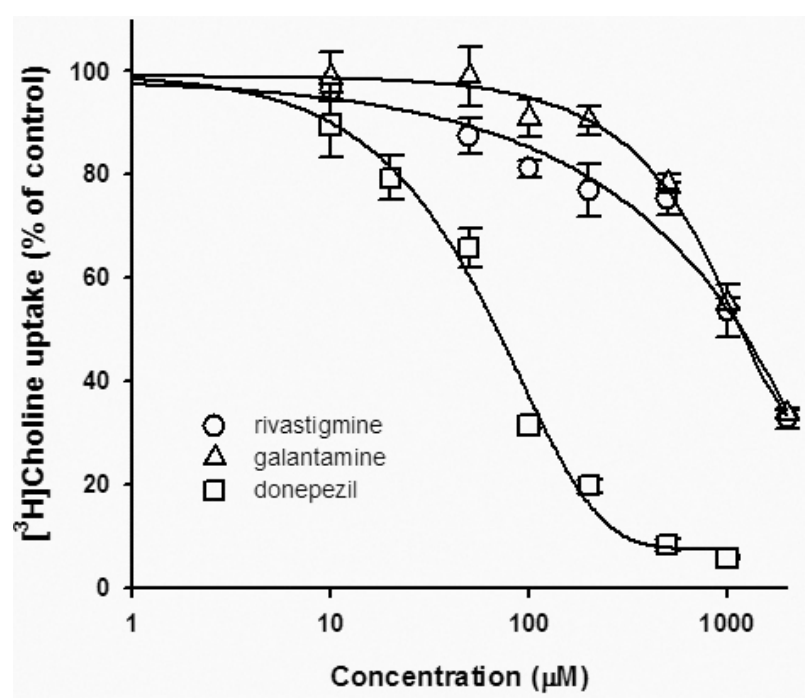

Fig. 1. Dose-response relationship for the inhibition of $\left[{ }^{3} \mathrm{H}\right]$ choline uptake by rivastigmine, galantamine and donepezil. Uptake of $\left[{ }^{3} \mathrm{H}\right]$ choline was measured in TR-BBB cells with $5 \mathrm{~min}$ incubation in the presence of $0-2,000 \mu \mathrm{M}$ unlabeled rivastigmine (open circle), galantamine (open triangle) and donepezil (open square) at $\mathrm{pH} 7.4$ and $37^{\circ} \mathrm{C}$. The data represent the mean \pm S.E.M. $(n=3-4)$.

Table II. $I \mathrm{C}_{50}$ and $K_{\mathrm{i}}$ values of donepezil, rivastigmine and galantamine for choline uptake in TR-BBB cells

\begin{tabular}{lcc}
\hline AChE inhibitor & $\mathrm{IC}_{50}(\mathrm{mM})$ & $K_{\mathrm{i}}(\mathrm{mM})$ \\
\hline Donepezil & 0.068 & 0.046 \\
Rivastigmine & 1.13 & 1.01 \\
Galantamine & 1.15 & None \\
\hline
\end{tabular}

(A)

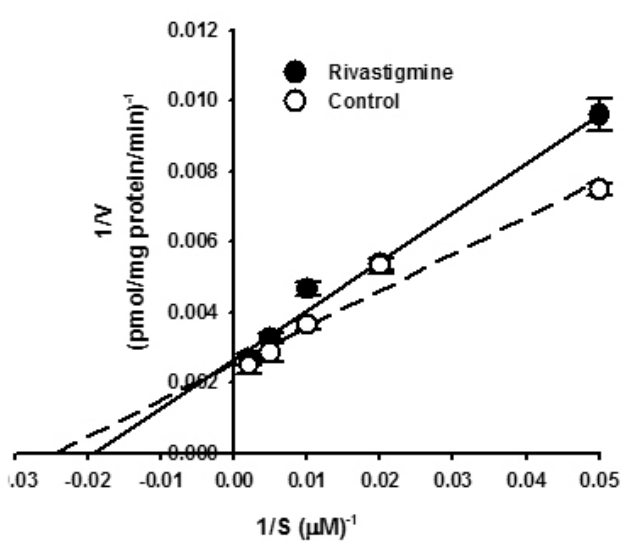

(B)

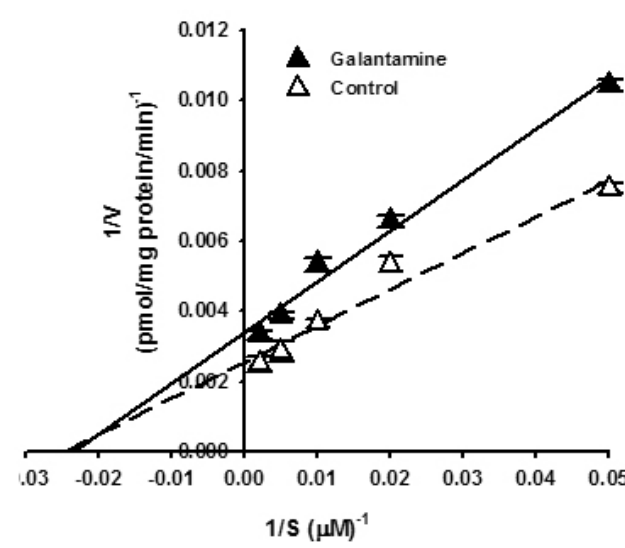

Fig. 2. Lineweaver-Burk plots of $\left.{ }^{3} \mathrm{H}\right]$ choline uptake by TR-BBB cells showing competitive or noncompetitive inhibition by rivastigmine $(A)$ and galantamine $(B)$. $\left[{ }^{3} \mathrm{H}\right]$ choline $(28 \mathrm{nM})$ uptake was performed in the presence of $500 \mu \mathrm{M}$ rivastigmine (closed circle), $500 \mu \mathrm{M}$ galantamine (closed triangle) or the absence (open circle, triangle) in TR-BBB cells at $\mathrm{pH} 7.4$ and $37^{\circ} \mathrm{C}$ for $5 \mathrm{~min}$. The data represents the mean \pm S.E.M. $(n=3-4)$. 


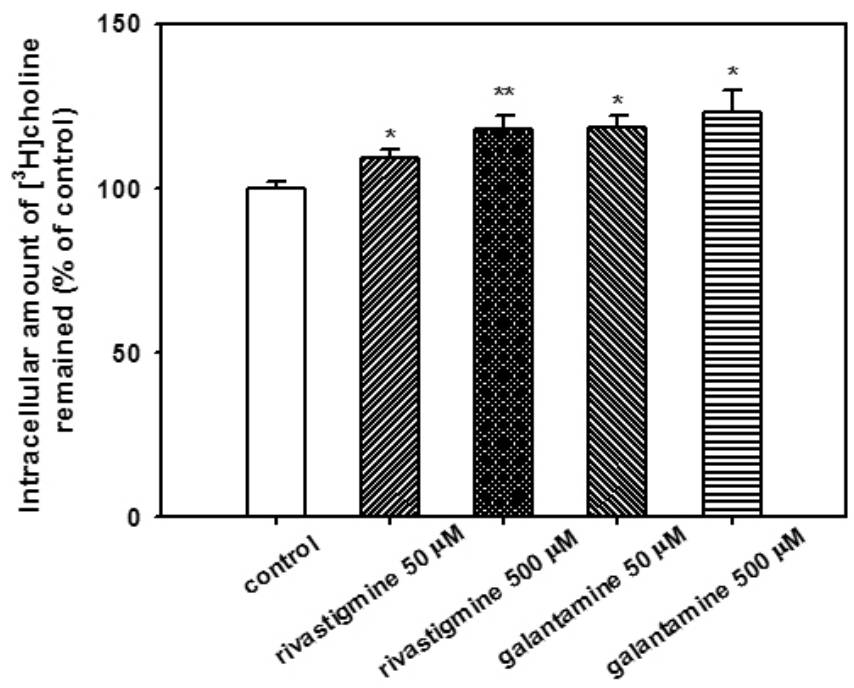

Fig. 3. Effect of rivastigmine and galantamine on efflux of $\left[{ }^{3} \mathrm{H}\right]$ choline in TR-BBB cells. TR-BBB cells were first incubated for 60 min at $37^{\circ} \mathrm{C}$ with ECF buffer containing $\left[{ }^{3} \mathrm{H}\right]$ choline $(28$ $\mathrm{nM})$. Then, the incubation medium alone or containing rivastigmine or galantamine was added for $30 \mathrm{~min}$ at $37^{\circ} \mathrm{C}$. After that, the incubation medium was removed and the amount of $\left[{ }^{3} \mathrm{H}\right]$ choline remained in the cells was measured. Each value represents the mean \pm S.E.M $(n=3) .{ }^{*} p<0.05,{ }^{* *} p<0.01$; significantly different from control.

$1.01 \mathrm{mM}$. In contrast, $K_{\mathrm{m}}$ value for choline uptake by TR-BBB cells did not change in the presence of $500 \mu \mathrm{M}$ galantamine. These results indicated that galantamine represent noncompetitive inhibition for choline uptake.

In addition, the effects of rivastigmine and galantamine on choline efflux transport in TR-BBB cells were studied. $\left[{ }^{3} \mathrm{H}\right]$ Choline efflux was inhibited significantly by rivastigmine and galantamines regardness of the drug concentrations in TR-BBB cells (Fig. 3).

\section{DISCUSSION}

In the present study, we investigated the interaction of rivastigmine and galantamine with choline transporter at the BBB using TR-BBB cells as an in vitro model of BBB. TR-BBB cells was established from transgenic rats harboring temperature-sensitive SV40 large T antigen gene, exhibited the fastest growth at $33^{\circ} \mathrm{C}$. These cells have been shown to express a number of receptors and transporters including glucose transporter-1 (GLUT-1), large neutral amino acid transporter (LAT1) and P-glycoprotein (P-gp) that are considered as specific for endothelial cells (Hosoya et al., 2000). Also, tight junction protein such as claudine-5 and occludin are also expressed in these cells (Hosoya et al., 2002). Further, the BBB permeability clearances of several compounds estimated in TR-BBB cells appear quite similar to in vivo evaluations (Terasaki et al., 2003). TR-BBB cells have already been used to demonstrate that transporters such as taurine transporter (TauT), system A isoform (ATA2) are expressed in rat brain endothelium (Kang et al., 2002; Takanaga et al., 2002). Accordingly, we thought that TR-BBB cells are one of the best in vitro models of BBB, allowing for the investigation of the mechanism for transport of diverse compounds including various drugs.

The BBB restricts the transport of cations from the blood to the brain parenchyma by a number of tight junctional proteins and tight junctions of the brain capillary endothelial cells (Pardridge, 1988). Despite these restrictions, it has been shown that the charged quaternary ammonium molecule, choline, is transported across the BBB via a saturable carrier-mediated mechanism (Cornford et al., 1978). The plasma choline concentration in healthy adults is approximately $10 \mu \mathrm{M}$ (Cohen et al., 1995), which is less than the calculated $K_{\mathrm{m}}$ for the BBB choline transporter (Sawada et al., 1999; Allen and Smith, 2001; Kang et al., 2005). This fact suggests that this transporter is not saturated under physiological condition and may deliver other compounds to the brain. Actually, the BBB choline transporter has been also reported to play an important role in the brain uptake of other organic cationic compounds such as eperisone, derivatives of lobeline, isoarecolone, and nicotine (Kang et al., 1990; Metting et al., 1998; Allen et al., 2003). Therefore, the BBB choline transporter has been suggested to be suitable as one of drug delivery vectors for cationic drugs.

AChE inhibitors such as donepezil, tacrine, rivastigmine and galantamine are used therapeutic agents for AD by preventing the destruction of synaptic acetylcholine. Among representative AChE inhibitors, donepezil and tacrine have been known to be transported to the brain across the BBB by choline transport system (Kang et al., 2005; Sung et al., 2005; Kim et al., 2009). However, the transport mechanism(s) of rivastigmine and galantamine remains unclear. It has been reported that these drugs cross the BBB and show their pharmacological action (Jann et al., 2002). Rivastigmine and galantamine are also organic cations in spite of having different molecular structures from donepezil and tacrine. Therefore, in this study, we hypothesized that BBB choline transporter may be also involved in transport of rivastigmine and galantamine across the BBB.

In the results of our study, $\left[{ }^{3} \mathrm{H}\right]$ choline uptake in the presence of rivastigmine and galantamine was decreased in a concentration dependent manner (Table I, Fig. 1), and 
the $\mathrm{IC}_{50}$ values for donepezil, rivastigmine and galantamine were $0.068,1.13$ and $1.15 \mathrm{mM}$, respectively (Table II). Also, rivastigmine competitively inhibited choline uptake with $K_{\mathrm{i}}$ of $1.01 \mathrm{mM}$, but galantamine represented noncompetitive inhibition for choline uptake in the LineweaverBurk plot analysis (Fig. 2). These results indicate that rivastigmine as well as donepezil compete with choline for the same binding site on the transporter. It was reported that the $K_{m}$ value for $\left[^{3} \mathrm{H}\right]$ choline uptake by TR-BBB cells were about $26 \mu \mathrm{M}$ (Kang et al., 2005). In the case of donepezil, the $K_{\mathrm{i}}$ values estimated for choline $(45.7 \mu \mathrm{M})$ was similar to $K_{\mathrm{m}}$ value for choline, suggesting the occurrence of competition between donepezil and choline for binding to the transporter like the results of previous reports. However, the affinity of rivastigmine for the choline transporter is relatively low compared with that of donepezil. Rivastigmine is a cabamate derivative and has a molecular weight of 400.43 and n-octanol to water partition coefficient of 3.0 (Polinsky, 1998). Based on the chemical properties, it would be expected that BBB transport of rivastigmine is carried out by passive diffusion (Murakami et al., 2000). However, our result proved that BBB choline transport system also involved in the transport of rivastigmine across the BBB. Taken together, we suggest that a part of rivastigmine penetrating into the brain following systemic administration is mediated by the BBB choline transporter and the remaining is the result of passive diffusion.

Choline is an essential nutrient that plays an important role as a precursor for the synthesis of the neurotransmitter acetylcholine (Cornford et al., 1978). Abnormal choline transport and metabolism have been implicated in a number of neurodegenerative disorders such as Alzheimer's and Parkinson's disease. In this respect, the BBB choline transporter is free to transport other cationic molecules without interrupting the supply of choline to the brain. The maximal plasma concentrations of donepezil and rivastigmine at the steady states were $61 \mathrm{ng} / \mathrm{ml}$ (Tiseo et al., 1998) and $56 \mathrm{ng} / \mathrm{ml}$ (Polinsky, 1998), respectively, and plasma protein binding rates of donepezil, rivastigmine and galantamine were $95 \%, 40 \%$ and $18 \%$, respectively (Jann et al., 2002). These data indicated that plasma concentrations of donepezil and rivastigmine were several times lower than $K_{i}$ value of them. Therefore, AChE inhibitors may not significantly inhibit choline transport at the BBB.

In addition, rivastigmine and galantamine also inhibited efflux transport of choline in TR-BBB cells (Fig. 3). In our previous report, it showed that choline underwent efflux from brain to blood across the BBB via a carrier-mediated efflux transport system which has the similar blood-to- brain influx rate (Lee and Kang, 2006). Therefore, this efflux system is supposed to play an important role in the choline turnover in the brain. Our previous report, donepezil and tacrine inhibited choline efflux via choline transport system in vivo and in vitro BBB model (Lee and Kang, 2008). Our result suggests that brain-to-blood efflux of rivastigmine and galantamine also occur by BBB choline transporter like donepezil and tacrine. These findings also suggest that these drugs may play a role in maintaining choline concentration in the brain resulting in the increase of acetylcholine synthesis.

In conclusion, it was found that the BBB choline transporter may be involved in a part of the influx and efflux transport of rivastigmine across the BBB. These findings also show that AChE inhibitors, rivastigmine as well as donepezil and tacrine, which are various organic cationic compounds, may exhibit increased brain distribution via the choline transport system. These findings should be therapeutically relevant to the treatment of $A D$ with $A C h E$ inhibitors, and, more generally, to the BBB transport of CNS-acting cationic drugs via choline transporter.

\section{ACKNOWLEDGMENTS}

The authors wish to thank $\mathrm{Dr}$. Terasaki for providing TR-BBB cell lines and Novartis International Pharmaceutical Ltd. for providing rivastigmine very kindly.

\section{REFERENCES}

Allen, D. D., Lockman, P. R., Roder, K. E., Dwoskin, L. P. and Crooks, P. A. (2003). Active transport of high-affinity choline and nicotine analogs into the central nervous system by the blood-brain barrier choline transporter. J. Pharmacol. Exp. Ther. 304, 1268-1274.

Allen, D. D. and Smith, Q. R. (2001). Characterization of the blood-brain barrier choline transporter using the in situ rat brain perfusion technique. J. Neurochem. 76, 1032-1041.

Cohen, B. M., Renshaw, P. F., Stoll, A. L., Wurtman, R. J., Yurgelun-Todd, D. and Babb, S. M. (1995). Decreased brain choline uptake in older adults: an in vivo proton magnetic resonance spectroscopy study. J. Am. Med. Assoc. 274, 902-907.

Cornford, E. M., Braun, L. D. and Oldendorf, W. H. (1978). Carrier mediated blood-brain barrier transport of choline and certain analogs. J. Neurochem. 30, 299-308.

Hosoya, K. I., Ohtsuki, S. and Terasaki, T. (2002). Recent advances in the brain-to-blood efflux transport across the blood-brain barrier. Int. J. Pharm. 248, 15-29.

Hosoya, K. I., Takashima, T., Tetsuka, K., Nagura, T., Ohtsuki, S., Takanaga, H., Ueda, M., Yanai, N., Obinata, M. and Terasaki, T. (2000). mRNA expression and transport characterization of conditionally immortalized rat brain capillary endothelial cell lines, a new in vitro BBB model for drug targeting. J. Drug Target. 8, 357-370. 
Jann, M. W., Shirley, K. L. and Small, G. W. (2002). Clinical pharmacokinetics and pharmacodynamics of cholinesterase inhibitors. Clin. Pharmacokinet. 41, 719-739.

Kang, Y. S., Terasaki, T., Ohnishi, T. and Tsuji, A. (1990). In vivo and in vitro evidence for a common carrier mediated transport of choline and basic drugs through the blood-brain barrier. J. Pharmacobiodyn. 13, 353-360.

Kang, Y. S., Lee, K. E., Lee, N. Y. and Terasaki, T. (2005). Donepezil, tacrine and alpha-phenyl-n-tert-butyl nitrone (PBN) inhibit choline transport by conditionally immortalized rat brain capillary endothelial cell lines (TR-BBB). Arch. Pharm. Res. 28, 443-450.

Kang, Y. S., Ohtsuki, S., Takanaga, H., Tomi, M., Hosoya, K. and Terasaki, T. (2002). Regulation of taurine transport at the blood-brain barrier by tumor necrosis factor-alpha, taurine and hypertonicity. J. Neurochem. 83, 1188-1195.

Kim, M. H., Maeng, H. J., Yu, K. H., Lee, K. R., Tsuruo, T., Kim, D. D., Shim, C. K. and Chung, S. J. (2009). Evidence of carrier-mediated transport in the penetration of donepezil into the rat brain. J. Pharm. Sci. 99, 1548-1566.

Lee, N. Y. and Kang, Y. S. (2006). In vivo brain-to-blood efflux transport of choline at the blood-brain barrier. J. Appl. Pharmacol. 14, 45-49.

Lee, N. Y. and Kang, Y. S. (2008). The efflux transport of choline through blood-brain barrier is inhibited by Alzheimer's disease therapeutics. Biomol. \& Ther. 16, 179-183.

Matsui, K., Mishima, M., Nagai, Y., Yuzuriha, T. and Yoshimura, T. (1999). Absorption, Distribution, Metabolism, and Excretion of Donepezil (Aricept) after a Single Oral Administration to Rat. Drug Metab. Dispos. 27, 1406-1414.

McNally, W. P., Pool, W. F., Sinz, M. W., Dehart, P., Ortwine, D. F., Huang, C. C., Chang, T. and Woolf, T. F. (1996). Distribution of tacrine and metabolites in rat brain and plasma after single- and multiple-dose regimens; Evidence for accumulation of tacrine in brain tissue. Drug Metab. Dispos. 24, 628-633.

Metting, T. L., Burgio, D. E., Terry, A. V., Beach, J. W., Mccurdy, C. R. and Allen, D. D. (1998). Inhibition of brain choline uptake by isoarecolone and lobeline derivatives: implications for potential vector-mediated brain drug delivery. Neurosci. Let. 258, 25-28.

Murakami, H., Takanaga, H., Matsuo, H., Ohtani, H. and Sawada, Y. (2000). Comparison of blood-brain barrier permeability in mice and rats using in situ brain perfusion technique. Am. J. Physiol. Heart Circ. Physiol. 279, H10221028.

Ohtsuki, S. and Terasaki T. (2007). Contribution of carriermediated transport systems to the blood-brain barrier as a supporting and protecting interface for the brain; importance for CNS drug discovery and development. Pharm. Res. 24, 1745-1758.

Pardridge, W. M. (1988). Recent advances in blood-brain barrier transport. Annu. Rev. Pharmacol. Toxicol. 28, 25-39.

Polinsky, R. J. (1998). Clinical pharmacology of rivastigmine: a new-generation acetylcholinesterase inhibitor for the treatment of Alzheimer's disease. Clin. Ther. 20, 634-647.

Sawada, N., Takanaga, H., Matsuo, H., Naito, M., Tsuruo, T. and Sawada, Y. (1999). Choline uptake by mouse brain capillary endothelial cells in culture. J. Pharm. Pharmacol. 51, 847-852.

Sung, J. H., Yu, K. H., Park, J. S., Tsuruo, T., Kim, D. D., Shim, C. K., Chung, S. J. (2005). Saturable distribution of tacrine into the striatal extracellular fluid of the rat: evidence of involvement of multiple organic cation transporters in the transport. Drug. Metab. Dispos. 33, 440-448.

Takanaga, H., Tokuda, N., Ohtsuki, S., Hosoya, K. and Terasaki, T. (2002). ATA2 is predominantly expressed as system $A$ at the blood-brain barrier and acts as brain-toblood efflux transport for L-proline. Mol. Pharmacol. 61, 1289-1296.

Terasaki, T., Ohtsuki, S., Hori, S., Takanaga, H., Nakashima, E. and Hosoya, K. (2003). New approaches to in vitro models of blood-brain barrier drug transport. Drug Discov. Today. 8, 944-954.

Tiseo, P. J., Rogers, S. L. and Friedhoff, L. T. (1998). Pharmacokinetic and pharmacodynamic profile of donepezil $\mathrm{HCl}$ following evening administration. Br. J. Clin. Pharmacol. 46, 13-18. 